Necrotising lymphadenitis without granulocytic infiltration: case from Western Samoa

Kikuchi's disease (necrotising lymphadenitis) was first reported by $\mathrm{Kikuchi}^{1}$ and Fujimoto et $a l^{2}$ in Japan in 1972. It is characterised by cervical lymphadenitis in young patients, and may simulate malignancy both clinically and histopathologically. Patients present with tender cervical or submandibular lymphadenopathy for a couple of weeks. There are generally no other abnormal physical signs and laboratory findings. The principal microscopical features are effacement of the lymph node architecture, coagulative necrosis to a varying degree, and infiltration of histiocytic cells without polymorphs; coagulative necrosis with an absence of polymorphs is the most striking histopathological finding of this disease.

Since 1972 cases outside Japan have also been reported in North America, the Far East, and the United Kingdom. ${ }^{3}$ This report concerns a case of Kikuchi's disease encountered in the island of Western Samoa believed to be the first such case report in that country.

A 25 year old Samoan (Polynesian) woman presented with left cervical and axillary lymphadenopathy which she had had for a few months. She first developed tender left cervical adenopathy, and later a painful swelling in the left axillary. Routine radiological and laboratory investigations showed no other important findings. A Paul-Bunnell test

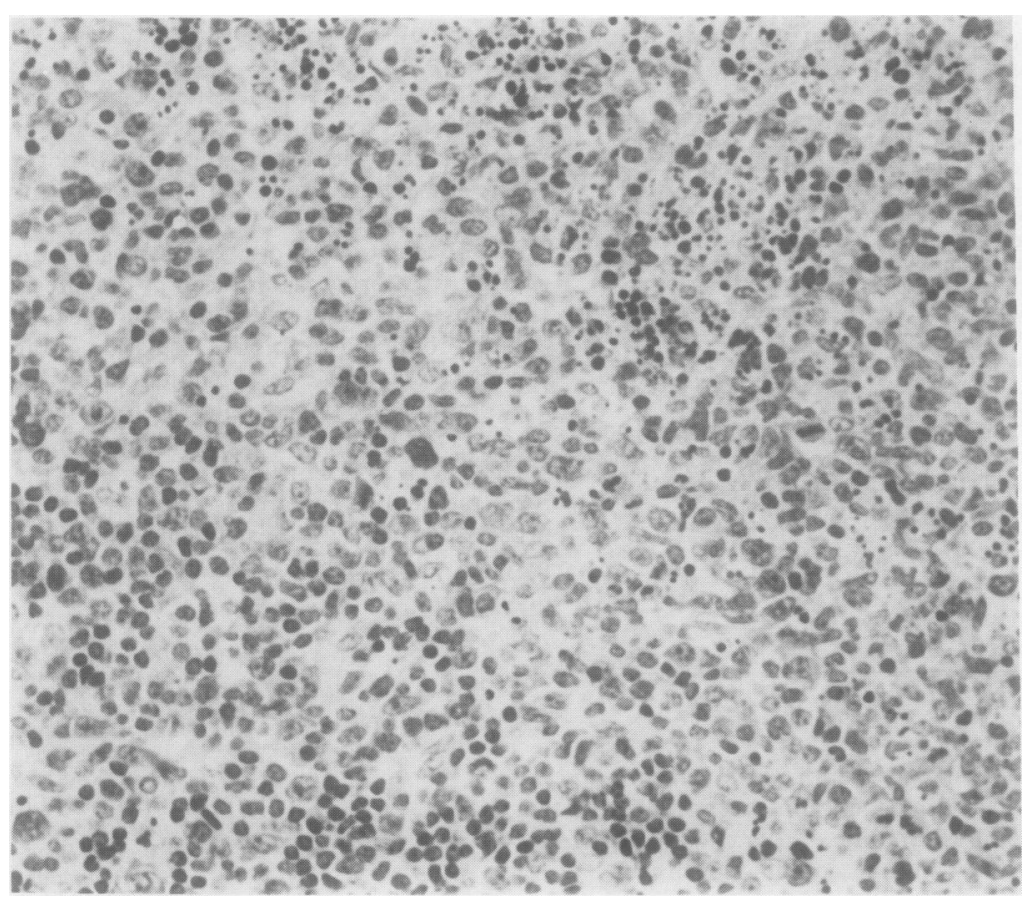

Lymph node biopsy specimens showing necrotic foci and histiocytic cells with phagocyic debris (haematoxylin and eosin).

\section{Fibrinogen standards}

In our laboratory fibrinogen concentrations are determined by the method of Clauss using a semiautomated technique (FibroSystem BBL; Becton Dickinson). The precision of our method is high (coefficient of variation of replicate samples $<4 \%$ ). Recently, however, we have noticed a discrepancy between results obtained when

was negative. Finally, the cervical node was biopsied with the presumptive diagnosis of tuberculosis.

Macroscpically the node was $2 \mathrm{~cm}$ in diameter, hard in consistency, with a greywhitish cut surface. Microscopically the node showed essentially similar features to those mentioned above: complete loss of the nodal architecture with varying degrees of necrosis, nuclear debris, and phagocytic histiocytic cells (figure). There was no neutrophil polymorph infiltrate. Acid fast stain was negative, as was bacteriological culture for tuberculosis. After consultation with the Department of Anatomical Pathology of the Sydney Royal Prince Alfred Hospital, Australia (Dr S McCarthy) the appearances were thought to be those of Kikuchi's disease. The disease is thought to be benign; the aetiology is unknown, although a viral aetiology has been suggested.

N PAKSOY
Department of Pathology,
School of Medicine,
PK 351, Merkez postane,
Antalya, Turkey

1 Kikuchi M. Lymphadenitis showed focal reticulum cell hyperplasia with nuclear debri and phagocytosis. Acta Haematol Jpn 1972, 35:379-80.

2 Fujimoto Y, Kozima Y, Yamaguchi K. Cervical subacute necrotising lymphadenitis. A new clinicopathological entity. Arch Intern Med 1972;30:920-7.

3 Ali MH, Horton LWL. Necrotising lymphadenitis without granulocytic infiltration (Kikuchi's disease). J Clin Pathol 1985; 38:1252-7. 